\title{
Developing DNA vaccines that call to dendritic cells
}

\author{
Michele A. Kutzler and David B. Weiner
}

Department of Pathology and Laboratory Medicine, University of Pennsylvania School of Medicine, Philadelphia, Pennsylvania, USA.

\begin{abstract}
DNA vaccination is a novel immunization strategy that has great potential for the development of vaccines and immune therapeutics. This strategy has been highly effective in mice, while less immunogenic in nonhuman primates and humans. Enhancing DNA vaccine potency remains a challenge. It is likely that APCs, and especially DCs, play a paramount role in the presentation of vaccine antigen to the immune system. A new study reports the synergistic recruitment, expansion, and activation of DCs in vivo in a mouse model through covaccination with plasmids encoding macrophage inflammatory protein-1 $\alpha$ (MIP-1 $\alpha)$, fms-like tyrosine kinase 3 ligand (Flt3L), and the DNA vaccine (see the related article beginning on page 1334). Such cooperative strategies delivering vaccine in a single, simple platform result in improved cellular immunity in vivo, including enhanced tetramer responses and IFN- $\gamma$ secretion by antigen-specific cells.
\end{abstract}

In theory, DNA vaccination combines the most desirable attributes of standard vaccine approaches in order to achieve functional enhancement of antigen-specific immune cells. Attenuated vaccine antigens are poorly taken up by and processed through the MHC class I pathway and thus primarily induce humoral immune responses and only weakly activate cytotoxic $\mathrm{T}$ cells. In contrast, live attenuated vaccines generate both cellular and humoral immune responses, and, potentially, lifelong immunity. However, safety concerns make development of new vaccines based on live, replicating vectors costly. Possible reversion and possible pathogenesis of the immunogen in immunocompromised individuals limit development of some live attenuated vaccines. The development of safer and more efficacious vaccine strategies that elicit both strong cellular and strong humoral immunity without viral replication is currently under intense investigation. One possible solution is the development of more effective DNA vaccines.

Nonstandard abbreviations used: CCR, CC chemokine receptor; env, envelope; Flt3L, fms-like tyrosine kinase 3 ligand; MIP- $1 \alpha$, macrophage inflammatory protein- $1 \alpha$.

Conflict of interest: The authors have declared that no conflict of interest exists.

Citation for this article: J. Clin. Invest. 114:1241-1244 (2004). doi:10.1172/JCI200423467.

\section{What are the mechanisms of immunity following DNA vaccination?}

The efficiency of DNA vaccination depends on the interaction among genetic material, lymphocytes, and APCs. Following intramuscular injection, myocytes play the role of antigen factories. They primarily express MHC class I and under certain circumstances express MHC class II; however, they do not express the costimulatory molecules required for priming and activation of $\mathrm{T}$ cells and thus lack function as effective APCs (1). Alternatively, small numbers of bone marrow-derived DCs and other professional APCs that can be activated to express high levels of MHC, as well as costimulatory molecules, are present at the site of injection and become transfected with the injected DNA. Cross-priming may occur, in which $\mathrm{CD}^{+} \mathrm{T}$ cell responses are primed by exogenous class I-restricted peptides that are not expressed in, but rather are acquired by, local APCs $(2,3)$. Regardless of the underlying mechanism, it has become clear that in the context of DNA vaccination APCs are key inducers of immunity, as they are the pivotal mediators of immune responses between resident somatic cells and $T$ cells in the lymph nodes. By trafficking antigen from the site of injection to the secondary lymphoid organs, APCs serve to efficiently present antigen to naive $T$ cells.

\section{Targeting DC maturation for} improved DNA vaccine potency

Based on the initial reports of immune enhancement by delivery of plasmids encoding GM-CSF (4) or IL-12 (5) in combination with specific antigen-encoded plasmids, other molecules have been studied for manipulating the potency of DNA vaccines in mice. Molecules under investigation target Th1-type T cell expansion; DC/APC activation, expansion, or maturation; costimulation; and immune cell trafficking by chemokines (4, 6-13). Recently, some vaccine strategies have produced exciting results in nonhuman primates $(14,15)$.

The ability of DCs to drive antigenspecific immunity is dependent on their degree of maturation. As shown in Table 1 , a variety of molecules delivered as DNA vaccines can improve APC activation, expansion, or maturation following antigen uptake and processing in vivo. These include bacterially derived antigens, TNFsuperfamily receptors, growth factors, inflammatory cytokines/chemokines, ligation of select cell surface receptors (CD40), and viral products. During their conversion from immature to mature cells, DCs undergo a number of phenotypic and functional changes, including increased MHC complex expression on their surface; increased surface expression of costimulatory molecules; morphological changes, including an increase in surface area, which allows DCs to interact with a greater number of $\mathrm{T}$ cells; secretion of chemokines and cytokines for attraction and expansion of T cells; protease production to convert molecules important in immune expansion to their active forms; and surface expression of adhesion molecules and chemokine receptors, allowing for increased interaction with other immune cells (Table 1). Through the use of combination immunomodulatory adjuvants that code for maturation, activation, or recruitment factors, it should be possible to further manipulate APCs in vivo to enhance DNA vaccine potency. 
Table 1

Molecular interactions that contribute to the recruitment, activation, or maturation of DCs in DNA vaccine studies

\section{DC receptor Expressed on immature/ Major ligand} mature DCs

DC effect

$\begin{array}{lc}\text { Chemokine receptor } & \\ \text { CCR1 } & \text { Immature } \\ \text { CCR2 } & \text { Immature } \\ \text { CCR5 } & \text { Immature } \\ \text { CCR6 } & \text { Immature } \\ \text { CXCR1 } & \text { Immature } \\ \text { CCR7 } & \text { Mature } \\ \text { CXCR4 } & \text { Mature }\end{array}$

Toll-like receptor

TLR2

TLR3
TLR9

Immature

Immature Immature

TNF-family receptor

$\begin{array}{lcccc}\text { Fas } & \text { Immature } & \text { FasL } & \text { Activation and maturation } & \text { Yes } \\ \text { CD40 } & \text { Immature } & \text { CD40L } & \text { Activation and maturation } & \text { Yes } \\ \text { OX40L } & \text { Mature } & \text { OX40 } & \text { Activation } & \text { Yes } \\ & & & \\ & \text { Growth factors } & \\ & \text { Flt3L } & \text { Expansion and activation } & \\ & \text { GM-CSF } & \text { Activation and maturation } & \\ & \text { TNF- } \alpha & \text { Maturation }\end{array}$

CpGs, oligos containing unmethylated cytosine and guanidine motifs; CXCR, CXC chemokine receptor; GCP-2, granulocyte chemotactic protein-2; HSP70, heat shock protein-70; NAP-2, neutrophil-activating peptide 2; OX40, TNF superfamily 4; OX40L, OX40 ligand, TNF receptor superfamily 4; SDF, stromal-derived factor; SLC, secondary lymphoid-tissue chemokine; TLR, toll-like receptor.

In this issue of the JCI, Sumida et al. (16) explore a combination of DC-specific chemotactic and growth factors, macrophage inflammatory protein- $1 \alpha$ (MIP-1 $\alpha$ ) and fms-like tyrosine kinase 3 ligand (Flt $3 \mathrm{~L})$, as part of a DNA vaccine cocktail. Previous DNA vaccine studies have shown that MIP- $1 \alpha$, which binds to CC chemokine receptor 5 (CCR5) on immature DCs, is able to recruit DCs to the site of inoculation in mice, resulting in enhanced cellular immune responses and increased antibody titers (17). In other studies, the use of maturation factors or DC growth factors such as Flt3L immunoadjuvants has been shown to expand DC numbers in both mice and humans $(7,18)$. However, the combination of MIP-1 $\alpha$ and Flt3L tested by Sumida et al. (16) extends those early studies by demonstrating DC recruitment to the site of injection and migration of loaded APCs to the regional lymph nodes (16). Immunohistochemistry of injected muscle tissue shows that inoculation with plasmids encoding MIP- $1 \alpha$ and Flt $3 \mathrm{~L}$ with HIV-1 envelope (env) DNA vaccine resulted in recruitment of DCs that express CD11b and the activation markers CD83 and S100, as well as the maturation markers MHC class II and CD80. In this model, DCs that traffic to the inoculation site in response to MIP- $1 \alpha$ expression and contact Flt $3 \mathrm{~L}$ expressed on the muscle cells expand and mature in response to the growth factor Flt3L (Figure 1). Upregulation of CCR7 and loss of CCR5 on the mature DCs will cause them to traffic to the draining lymph node, where immune priming can occur in the germinal centers (Figure 1).

In the current study (16), vaccine-elicited immune responses were not detectably augmented by plasmid-encoded Flt3L or plasmid-encoded MIP- $1 \alpha$ alone. In contrast, tetramer responses and IFN- $\gamma$ responses were enhanced in mice that received the DNA vaccine along with both adjuvant plasmids. This finding is in contrast to earlier studies in which vaccination with MIP-1 $\alpha$ expanded both humoral and cellular immune responses (17) and vaccination with Flt3L also exhibited enhancement of immunogenicity (18). These differences may reflect the properties of different plasmid-based vectors, properties unique to the antigens involved, different forms of the adjuvants, or other, unknown biases.

Furthermore, improved protection against challenge with recombinant HIV env and vaccinia virus in mice was achieved following vaccination with plasmidencoded MIP-1 $\alpha$, Flt 3 L, and HIV-1 env DNA vaccine (16). Prior studies have also shown enhanced immunogenicity and protection against lethal challenge with herpes simplex virus- 2 in mouse model systems using chemokines that bind CCR5 as adjuvants. For example, RANTES coimmunization significantly enhanced $\mathrm{CD}^{+}$and $\mathrm{CD}^{+} \mathrm{T}$ cell responses and resulted in enhanced survival in a lethal mucosal herpes simplex virus- 2 challenge model (19). However, in the same system, immunization with MIP- $1 \alpha$ alone was not effective, which suggests that not all CCR5 ligands are similar and that Flt3L, as reported here (16), complements MIP- $1 \alpha$ activity.

Overall, these studies support a model where the number of DCs present at the site of inoculation during antigen expression is a possible rate-limiting factor for DNA vaccine effectiveness. In this context, coimmunization with a plasmid chemokine for attracting DCs to the site of injection appears important. For example, in other DNA vaccine studies, the use of apoptotic molecules as plasmid adjuvants results in the death of antigen-bearing cells, leading to an increase in antigen acquisition by APCs, including DCs $(20,21)$.

Important issues that should be investigated include elucidation of how the antigenic plasmid is trafficked to APCs following immunization, the efficacy of different adjuvant combinations, the most relevant timing of adjuvant delivery, and validation using other antigenic plasmids in additional model systems. The question remains of exactly how and in what context DCs that migrate to the site of injection acquire plasma-delivered antigen. For future development of this strategy, validation needs to be carried out in primate models. Many vaccine studies have shown that mice and primates may not respond similarly to the same adjuvants. For example, GM-CSF was shown to be a superior adjuvant for DNA vaccines in mice but was a poor DNA adju- 
A

Intramuscular immunization
B

DC recruitment to site of MIP- $1 \alpha$ immunization
C

Germinal centers

in secondary lymph organs

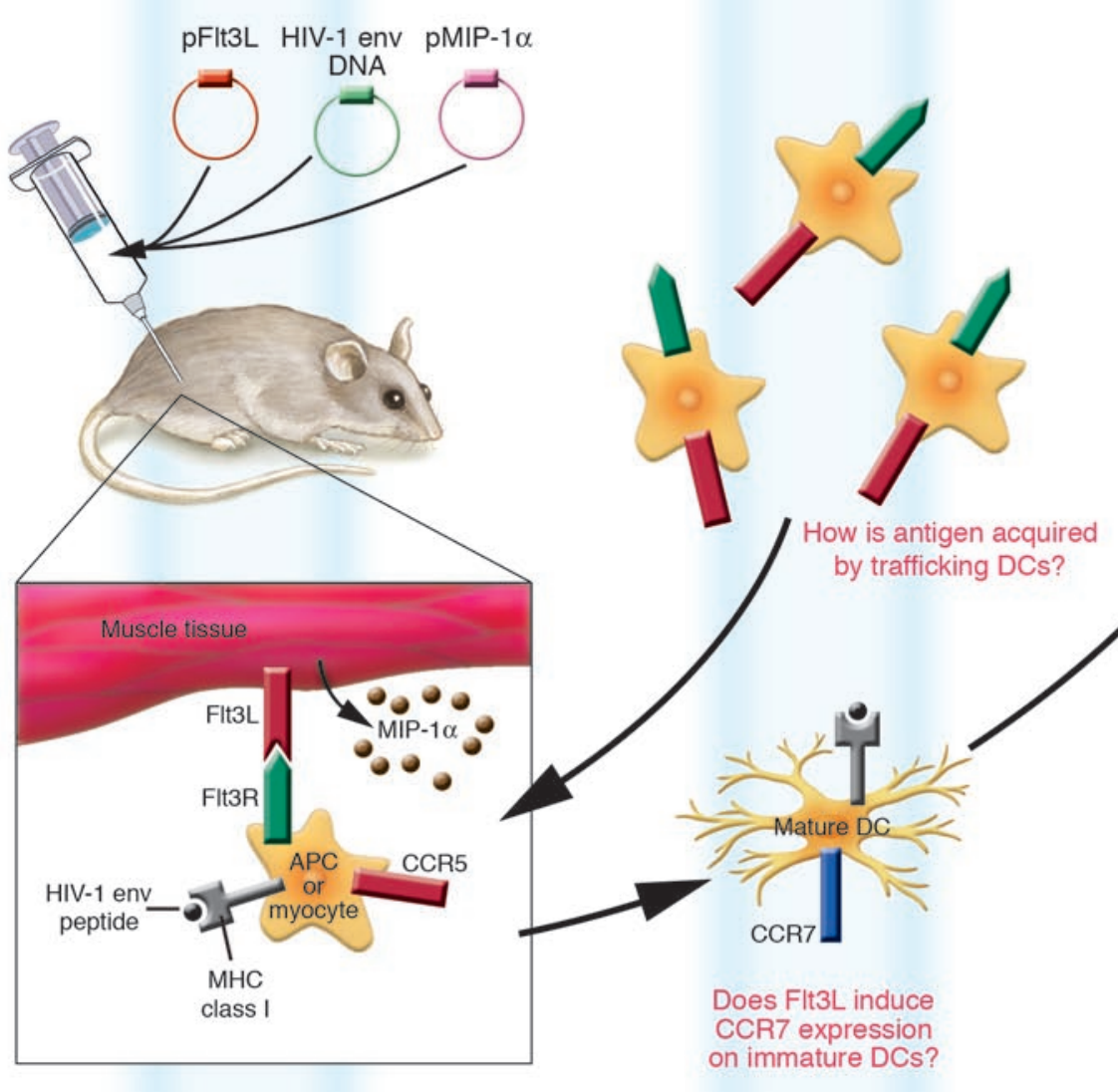

- MHC class II

- cytokines

- chemokines

- chemokine receptors

- costimulatory molecules (eg., CD80)

- activation markers

(eg., CD83, S100)

(ii) Morphological changes: - dendrite formation

\section{Figure 1}

Proposed schematic of chemokine-induced traffic and activation of DCs following DNA vaccination with plasmid-encoded FIt3L and MIP-1 $\alpha$. (A) Following intramuscular injection in mice of plasmid-encoded MIP-1 $\alpha$ (pMIP-1 $\alpha$ ) and plasmid-encoded Flt3L (pFlt3L), in combination with HIV-1 env, muscle and resident APCs are transfected with plasmid, leading to protein production. MIP-1 $\alpha$ protein is secreted, while Flt3L is expressed on muscle cells or on the surface of resident APCs. HIV-1 env is processed, and peptides are presented by MHC class I molecules; this peptide/MHC complex stimulates CD8+ T lymphocytes. Soluble protein released by transfected cells is taken up by APCs, and via the MHC Class II pathway, the peptide/MHC complex stimulates CD4+ $\mathrm{T}$ lymphocytes. Cross-priming occurs in which CD8+ ${ }^{+}$cell responses are primed by exogenous class I-restricted antigens that are not expressed in, but rather are acquired by, local APCs. (B) DCs that express CCR5 are recruited to the site of immunization in response to MIP- $1 \alpha$ secretion. DCs bind Flt3L on the surface of local APCs or muscle cells, resulting in their expansion and maturation as they migrate to the draining lymph node. How antigen is acquired by the newly recruited DCs is an important question that remains poorly understood. These DCs acquire antigen, express CCR7, and are attracted by chemokines expressed in the draining lymph nodes. (C) The same DCs as in B traffic to the draining lymph nodes, where they prime naive T cells. Mature DCs express high levels of MHC class II, costimulatory molecules, activation markers, adhesion molecules, and chemokine receptors, secrete cytokines and chemokines, and form dendrites.

vant in nonhuman primates (15). Such validation studies are an important step since the immune response induced in nonhuman primates appears to be more representative of the responses ultimately observed in humans. Sumida et al. (16) provide additional evidence that simple DNA vaccine formulations can manipu- late complex host immunity. A more detailed study of these and other combination strategies aimed at improved APC function will likely represent an important area of DNA vaccine research. Such combination strategies may ultimately allow this novel approach to achieve improved potency in clinical evaluation.
Address correspondence to: David Weiner, Department of Pathology and Laboratory Medicine, University of Pennsylvania School of Medicine, 505 Stellar Chance Laboratories, 422 Curie Boulevard, Philadelphia, Pennsylvania 19104, USA. Phone: (215) 662-2352; Fax: (215) 573-9436; E-mail: dbweiner@mail.med.upenn.edu. 
1. Hohlfeld, R., and Engel, A.G. 1994. The immunobiology of muscle. Immunol. Today. 15:269-274.

2. Heath, W.R., and Carbone, F.R. 2001. Cross-presentation in viral immunity and self-tolerance. Nat. Rev. Immunol. 1:126-134.

3. Ulmer, J.B., and Otten, G.R. 2000. Priming of CTL responses by DNA vaccines: direct transfection of antigen presenting cells versus cross-priming. Dev. Biol. (Basel). 104:9-14.

4. Xiang, Z., and Ertl, H.C. 1995. Manipulation of the immune response to a plasmid-encoded viral antigen by coinoculation with plasmids expressing cytokines. Immunity. 2:129-135.

5. Kim, J.J., et al. 1997. In vivo engineering of a cellular immune response by coadministration of IL-12 expression vector with a DNA immunogen. J. Immunol. 158:816-826.

6. Gurunathan, S., Wu, C.Y., Freidag, B.L., and Seder, R.A. 2000. DNA vaccines: a key for inducing longterm cellular immunity. Curr. Opin. Immunol. 12:442-447.

7. Chan, K., et al. 2001. The roles of MHC class II, CD40, and B7 costimulation in CTL induction by plasmid DNA. J. Immunol. 166:3061-3066.

8. Shedlock, D.J., and Weiner, D.B. 2000. DNA vaccination: antigen presentation and the induction of immunity. J. Leukoc. Biol. 68:793-806.
9. Kim, J.J., Yang, J.S., Dentchev, T., Dang, K., and Weiner, D.B. 2000. Chemokine gene adjuvants can modulate immune responses induced by DNA vaccines. J. Interferon Cytokine Res. 20:487-498.

10. Pasquini, S., et al. 1997. Cytokines and costimulatory molecules as genetic adjuvants. Immunol. Cell Biol. 75:397-401.

11. Biragyn, A., et al. 2002. DNA vaccines encoding human immunodeficiency virus-1 glycoprotein 120 fusions with proinflammatory chemoattractants induce systemic and mucosal immune responses. Blood. 100:1153-1159.

12. Spies, B., et al. 2003. Vaccination with plasmid DNA activates dendritic cells via Toll-like receptor 9 (TLR9) but functions in TLR9-deficient mice. J. Immunol. 171:5908-5912.

13. Wan, T., et al. 2004. Novel heat shock protein Hsp70L1 activates dendritic cells and acts as a Th1 polarizing adjuvant. Blood. 103:1747-1754.

14. Barouch, D.H., et al. 2000. Control of viremia and prevention of clinical AIDS in rhesus monkeys by cytokine-augmented DNA vaccination. Science. 290:486-492.

15. Calarota, S.A., and Weiner, D.B. 2004. Enhancement of human immunodeficiency virus type 1-DNA vaccine potency through incorporation of T-helper 1 molecular adjuvants. Immunol. Rev.
199:84-99.

16. Sumida, S.M., et al. 2004. Recruitment and expansion of dendritic cells in vivo potentiate the immunogenicity of plasmid DNA vaccines. J. Clin. Invest. 114:1334-1342. doi:10.1172/JCI200422608.

17. McKay, P.F., et al. 2004. Recruitment of different subsets of antigen-presenting cells selectively modulates DNA vaccine-elicited CD4+ and CD8+ T lymphocyte responses. Eur. J. Immunol. 34:1011-1020.

18. Westermann, J., et al. 2004. Flt-3 ligand as adjuvant for DNA vaccination augments immune responses but does not skew TH1/TH2 polarization. Gene Ther. 11:1048-1056

19. Sin, J., Kim, J.J., Pachuk, C., Satishchandran, C., and Weiner, D.B. 2000. DNA vaccines encoding interleukin-8 and RANTES enhance antigen-specific Th1-type CD4(+) T-cell-mediated protective immunity against herpes simplex virus type 2 in vivo. J. Virol. 74:11173-11180.

20. Chattergoon, M.A., et al. 2000. Targeted antigen delivery to antigen-presenting cells including dendritic cells by engineered Fas-mediated apoptosis. Nat. Biotechnol. 18:974-979.

21. Sasaki, S., Amara, R.R., Oran, A.E., Smith, J.M., and Robinson, H.L. 2001. Apoptosis-mediated enhancement of DNA-raised immune responses by mutant caspases. Nat. Biotechnol. 19:543-547.

\section{Do DNA sequence variants in $A B C A 1$ contribute to $\mathrm{HDL}$ cholesterol levels in the general population?}

Päivi Pajukanta

Department of Human Genetics, David Geffen School of Medicine at UCLA, University of California, Los Angeles, Los Angeles, California, USA.

\begin{abstract}
HDL has a key role in reverse cholesterol transport, mobilizing cholesterol from the peripheral tissues to liver. In this process, the $\mathrm{ABC}$ transporter $\mathrm{A} 1$ (ABCA1) protein controls the efflux of intracellular cholesterol to apoAI, the major apolipoprotein of HDL. Since $A B C A 1$ mutations were discovered to cause Tangier disease, a rare recessive HDL deficiency, it has been speculated that sequence variants in $A B C A 1$ might also contribute to variations in plasma HDL cholesterol levels in the general population. A new study provides genetic evidence supporting this hypothesis (see the related article beginning on page 1343).
\end{abstract}

A decreased level of plasma HDL cholesterol (HDL-C) is a major risk factor for coronary atherosclerosis. The cardioprotective effect of HDL has been attributed to, among other factors, its key role in reverse cholesterol transport (RCT), mobilizing cholesterol

Nonstandard abbreviations used: $\mathrm{ABCA} 1, \mathrm{ABC}$ transporter A1; APOA1, apolipoprotein AI; HDL-C, HDL cholesterol; LCAT, lecithin cholesterol acyltransferase; RCT, reverse cholesterol transport; SNP, single-nucleotide polymorphism.

Conflict of interest: The author has declared that no conflict of interest exists.

Citation for this article: J. Clin. Invest. 114:1244-1247 (2004). doi:10.1172/JCI200423466. from the peripheral tissues to liver. Approximately $50 \%$ of plasma HDL-C variability is determined by genetic factors $(1,2)$. Variants in several genes, including $A B C$ transporter A1 (ABCA1), apolipoprotein AI (APOA1), and lecithin cholesterol acyltransferase (LCAT), are implicated in rare mendelian forms of HDL deficiency (refs. 3-5; reviewed in refs. 6, 7). Several chromosomal regions have been identified in genome-wide scans for HDL-C, and these regions are likely to harbor genes for common forms of HDL-C deficiency variants of which have been shown to affect plasma HDL-C levels, include, for example, (8-13). In addition, candidate genes, the hepatic lipase and the apolipoprotein AI/CIII/ $A I V / A V$ gene cluster (14). However, DNA sequence variants contributing to variation in plasma levels of HDL-C in the general population are largely unknown, especially regarding the prevalence of variants with major effects on HDL-C level.

Generally, complex traits are suggested to be caused by common sequence variants that each may have a small to moderate phenotypic effect (15-17). On the other hand, accumulating data show that most mendelian disorders are caused by a set of different mutations that often reside in coding regions (reviewed in ref. 18). These rare variants tend to have strong phenotypic effects. The extent to which rare versus common variants confer the susceptibility to complex traits is currently not known. Studies such as that featured in this issue of the JCI by Frikke-Schmidt and colleagues (19), investigating whether rare and/or common variants contribute to a quantitative trait, are of major importance not only in elucidating the sequence variants 